This paper was presented at the 8 th International Conference on Agents and Artificial Intelligence, held in Rome, Italy on 24-26 Febrauary 2016, http: / www. icaart.org/ ?y= 2016.

Copyright of the contribution is owned by the publisher, Science and Technology Publications (SCITEPRESS), http://www.scitepress.org.

This material is presented to ensure timely dissemination of scholarly and technical work. All persons copying this information are expected to adhere to the terms and constraints invoked by relevant copyright. In most cases, this material may not be reposted without the explicit permission of the copyright holder.

Celentano, Ulrico; Röning, Juha (2016) Multi-robot systems, machine-machine and human-machine interaction, and their modelling. 8th International Conference on Agents and Artificial Intelligence (ICAART), vol. 1, pp. 118-125. 24-26 Feb, Rome, Italy. doi:10.5220/0005667801180125.

URL: http://www.scitepress.org/DigitalLibrary/Link.aspx?doi=10.5220/ 0005667801180125 


\title{
Multi-robot Systems, Machine-Machine and Human-Machine Interaction, and Their Modelling
}

\author{
Ulrico Celentano $^{1}$ and Juha Röning ${ }^{1}$ \\ ${ }^{1}$ Biomimetics and Intelligent Systems Group, Faculty of Information Technology and Electrical Engineering, University of \\ Oulu, P.O. Box 4500, FI-90014 Oulu, Finland \\ \{ulrico.celentano, juha.roning\}@ee.oulu.fi
}

\begin{abstract}
Keywords: Cognition and Metacognition, Cognitive Agents, Interworking Cognitive Entities, Multi-robot Systems, Social Interaction.

Abstract: The control of multi-agent systems, including multi-robot systems, requires some level of context and environment awareness as well as interaction among the interworked cognitive entities, whether they are artificial or natural. Proper specification of the cognitive functionalities and of the corresponding interfaces helps in achieving the capability to reach interoperability across different operational domains, and to reuse the system design across different application domains. The model for interworking cognitive entities presented in this article, which includes explicitly interworking capabilities, is applied to two major classes of interaction in multi-robot systems. Being the model inspired by both artificial and natural systems, makes it suitable for both machine-machine and human-machine interaction.
\end{abstract}

\section{INTRODUCTION}

The management or self-management of a multirobot system (MRS) requires interaction among all the involved interworked entities. At scattered entities, awareness of own environment and context is needed, and forms of distributed decision-making are possible. More, a MRS may realise various distributed sensor-actuator configurations since action at one entity may be based (also) on perceptions at other entities.

In a number of use cases, not only machinemachine but also human-machine interaction is present, hence both artificial and natural cognitive entities interact and their role as supervisor or as an object may change depending on use case or context. Embedding humans into the overall system allows taking into account their needs, capabilities and limitations, with an effect on the system architecture and the interfaces within the entire social ecosystem.

Discussion on the specification of the interfaces is needed because it may help interoperability of a system across operational domains and even in reusing system design across different application domains.

After having introduced in Sect. 3 multi-entity intelligent systems, this article starts building upon the identification in Sect. 4 of the issues related to the control of multi-robot systems as interworking cog- nitive entities and continues presenting in Sect. 5 a model for those constituent elements. For what seen above, a model should be as holistic as possible to encompass particularities of both artificial and natural cognitive entities. An example instantiation of the cognitive model in a multi-robot system scenario is illustrated in Sect. 6, whereas Sect. 7 discusses a comparative evaluation of the model. The core part in the above sections is preceded by a review of related work and other background in Sect. 2, and concluded by a discussion in Sect. 8.

\section{BACKGROUND}

Decision-making based on situational awareness requires interaction of multiple entities. Interoperability of informative systems is recognised as a crucial aspect in multinational, co-operative command and control (C2) operations, including peace-keeping. Related standardisation activity is promoted for example by the Simulation Interoperability Standards Organization (SISO) $)^{1}$. Interoperability is also one of the goal of the standardisation work done within the Third Generation Partnership Project (3GPP) to en-

\footnotetext{
${ }^{1}$ http://www. sisostds.org/. Last accessed 9 Sep 2015.
} 
able Long-Term Evolution (LTE) wireless communication system for public safety.

Cognitive processes - essentially involving perception, decision-making and action - are fundamental components in robot systems and human-machine interaction. Related models - see (Celentano, 2014; Celentano and Röning, ms) for a wider and deeper review - date back to years 1939 for quality control in industrial production (Shewhart, 1939), and 1945 for problem solving (Polya, 1957). These are both four-phase models: plan, do, check, act; and understand the problem, devise a plan, carry out the plan, look back; respectively. Models for robotics started also with three-four phase models - sense, (model), plan, act - (Nilsson, 1984) but got then radically revised by the introduction of the subsumption architecture, decomposing phases into elementary behaviours (Brooks, 1986). The adaptive control of thoughtrational (ACT-R) architecture was developed for humans but it was also applied to human-machine interaction (Anderson et al., 2004; Langley et al., 2009).

Specifically for C2 decision-making, John Boyd devised a model to understand adversaries - the observe-orient-decide-act (OODA) loop - further adapted to cognitive radios and extended (Thomas et al., 2005, e.g.) by Mitola (Mitola, 2000). A following proposal was the critique-explore-compare-adapt (CECA) model (Bryant, 2004). Its last three phases have similar duties as in the OODA model, but the critique makes the model more proactive. Whereas in the CECA model plan serves as a sort of initialisation phase before the context acquisition takes place, in (Mitola, 2000) it is used to take further actions as a consequence of the acquired context information. We may observe that OODA regulates the actions, whereas CECA regulates the plan.

The definition of a robot operating system dates back at least to 1984, with the control of a single robot but integrated into a system comprising sensors and humans (Dupourqué, 1984). Various operating systems (Kerr and Nickels, 2012) and architectures (Mäenpää et al., 2004, e.g.) have been proposed, with the open-source robot operating system ROS been recently used for multi-robot systems. ROS is developed for large-scale robot systems and is realised as a peer-to-peer topology of processes running on separate hosts and interconnected together (Quigley et al., 2009). ROS nodes communicate by messages. Although message structure can be customised, some message structures are encouraged to be adopted to enable interoperability.

A multi-robot system may be part of a larger ecosystem, possibly together with humans sometimes having a supervisory role, or embedded into the sys- tem with a peer role (Peschl et al., 2012). Under SISO, the battle management language (BML) has been designed as a human readable, unambiguous language to control robots integrated in an international warfare system, including interaction among command and control systems, human units and robots (Rein et al., 2009).

\section{MULTI-ENTITY INTELLIGENT SYSTEMS}

A generic model applicable to both artificial and natural entities is welcome since it is more easily applicable to systems in which both machines and humans are possibly present, see previous sections. Moreover, such a model also helps in more effectively bridging natural and artificial systems by exporting knowledge across these domains. A generic model is presented and discussed in this article, together with example applications of it.

Roughly, but see Sect. 5 and 7 for details, an intelligent system such as a robot or a human has three main functions: observation, decision-making and action execution. Many peculiarities and implications are involved in the action execution part. They include for example, in the case of robots, the practical actuation and the fine control of system dynamics. These aspects are left out of the scope of this article. On the contrary, central of interest in this article are decision-making and the necessary awareness derived from observations, see the illustrative examples in Sect. 4 and 6.

An important contribution of this article concerns the social interaction in multi-entity intelligent systems, see Sect. 5 and examples in Sect. 6.

Natural interfaces are created in a multi-entity cognitive system. For flexible and reusable design purposes it is an advantage to specify interfaces also for functions internal to an entity. This is facilitated by the specification of the duties of cognitive functions, see Sect. 5.

\section{CONTROL OF COGNITIVE MULTI-ROBOT SYSTEMS}

Multi-robot systems often require forms of cooperation, collaboration or co-ordination among the entities, such as collision-avoidance (Remmersmann et al., 2010) or flocking control (La et al., 2015), and for those inter-robot interaction is needed. Robots 
may possess various levels of cognition; see for example (Celentano, 2014).

The most straightforward way to implement the above forms of interaction is to let any entity to observe the actions (e.g., position and movement) of the other robots. Robots in this case sense the actuations of the other robots. Although in some cases prediction of actions (e.g. trajectories) of the other robots is possible, if tasks/plans of the other robots are unknown, or they are dynamically adapted (e.g. to avoid obstacles on the terrain), prediction may be impossible or inaccurate, see Fig. 1 and Fig. 2.

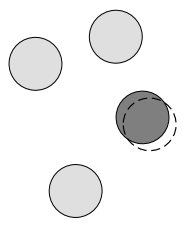

(a)

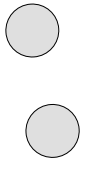

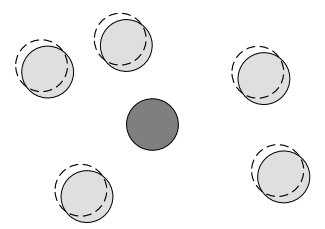

(b)
Figure 1: An example about self/non-self-awareness.

Fig. 1 illustrates an example about self/non-selfawareness. While the resulting relative configuration of the system is the same in both cases, on the left the displacement is caused by a movement of the marked robot (darker grey circle), and on the right it is due to the rest (lighter grey circles). The situation may be more complicated if not all of the lighter grey entities are synchronised. In any case, absolute positioning may be used in this example, but in general it may be quicker or more exact, or even feasible (absolute positioning may not always be available or it may not have the required resolution), deducting the cause from shared information.

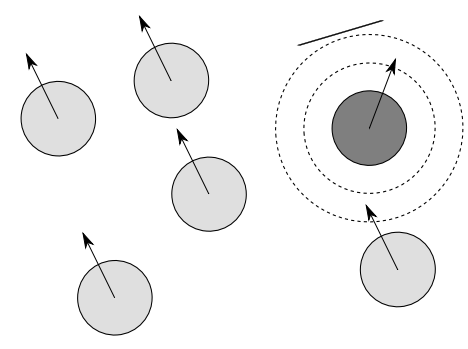

Figure 2: A fleet of robots with one avoiding an obstacle.

Fig. 2 shows a fleet of robots with one entity avoiding an obstacle. Despite the lead robot (darker grey circle) changes its course (to avoid an obstacle, straight solid line), the rest of the system (lighter grey circles) follows the originally agreed path, having been instructed (dashed circles) about the reason of the change.

The examples above are about managing behavioural anomalies (abnormalities in group dynam- ics), i.e., it is of interest to understand whether the reason for the anomaly is due to our entity or to the other. Similar decisions are needed for example in intervehicle coordination, which can be realised through warnings for man-controlled vehicles, or as part of their control, also applicable to unmanned vehicles. In decision making, explicit coordination can be exploited (for example by inter-entity communications, like in Fig. 2), or implicit information can be used (by self-awareness, like in the scenario of Fig. 1).

In general, three forms of interaction can be identified in an MRS: act-and-observe (robots observe the actions of other robots), share-and-act (robots act after negotiation of their actions), act-while-checking (robots behave initially as in the first case, but refine their operations as in the following case).

As noted, sharing part of robot's own plans is a way to improve coexistence within a multi-robot system. In this case, actions are based on exchange of information among robots, rather than observing their decisions (actions, movements). Indeed, misalignments due to own or other's errors require different responses; this means that self/nonself discrimination, discussed above, is needed.

From the above examples it is derived that robots may need to share their knowledge (and or plans).

\section{MODEL FOR COGNITION}

This section presents our model (Celentano, 2014) that introduces the required phases missing from those in the literature (Sect. 2 and references therein).

The specification of cognitive functionalities should respond to the needs outlined in Sect. 1. To this end, in our model presented here, the definition of functionalities - and as a consequence of the interfaces - is done in such a way that cognitive functions are neatly assigned to specific units or physical elements within it. The phases of the cognitive entity, i.e., the components instantaneously active in the cognitive process, are defined considering also their role in relation to the environment they operate within.

It is obvious that what is an action for one entity may be a perception for another. However, interaction of a cognitive entity with its environment is not limited to initial observation and final action events but it includes also interaction with intermediate cognitive phases in which more cognitive entities are involved. It is important to note that in general the interaction of the cognitive entities with their environment occurs during the cognitive process and not necessarily only at its start or end (i.e., at perception or action, respectively). This sharing of information, or knowledge, 
or sometimes commands - in this article, a piece of information or knowledge or a command are together referred to as instructions - takes possibly place before the actual actions are taken and therefore cannot be modelled as act-perceive interactions. New cognitive phases are needed for that.

Cognitive phases are divided into functionalities dealing with exchanging facts and implementing competences, and those in charge of processing knowledge, making decisions and learning. Metacognition is the knowledge about the cognitive phenomena (or knowledge of cognition) and the regulation of the cognition processes.

The phases of our model presented in this article are grouped under two layers:

- a cognitive frontier (represented by the outer circle in Fig. 3), in charge of interaction with external entities in the process of:

- acquiring (raw) information (sometimes referred to as data),

- storing and fetching accumulated knowledge,

- sharing instructions, and

- actuating decisions;

- a metacognitive hub (represented by the inner disc in Fig. 3), in charge of controlling cognitive phenomena and cognitive actions, i.e., of the interwork of the internal processes in:

- processing the acquired information,

- building knowledge from experience (both from outside information/knowledge and own decisions),

- preparing instructions, and

- generating the needed decisions.

The metacognitive hub generates a new level of information. For example, the correlation between the decide phase with the perceive for an endogenous stimulus to discriminate self/nonself happens near the boundary of the metacognitive hub.

Fig. 3 shows our model for a networked cognitive entity. Four categories span across two layers. Entity's input $\mathbf{u}$, output $\mathbf{y}$, internal state $\mathbf{y}$, stored knowledge $\mathbf{z}$ and shared instructions (information, knowledge or commands) $\mathbf{x}$ are also shown in the figure.

The phases introduced above belong to the following four categories (represented by the sectors in Fig. 3):

- observation (perceive, analyse),

- consolidation (learn, remember),

- interworking (synthesise, share) and

- operation (decide, apply).

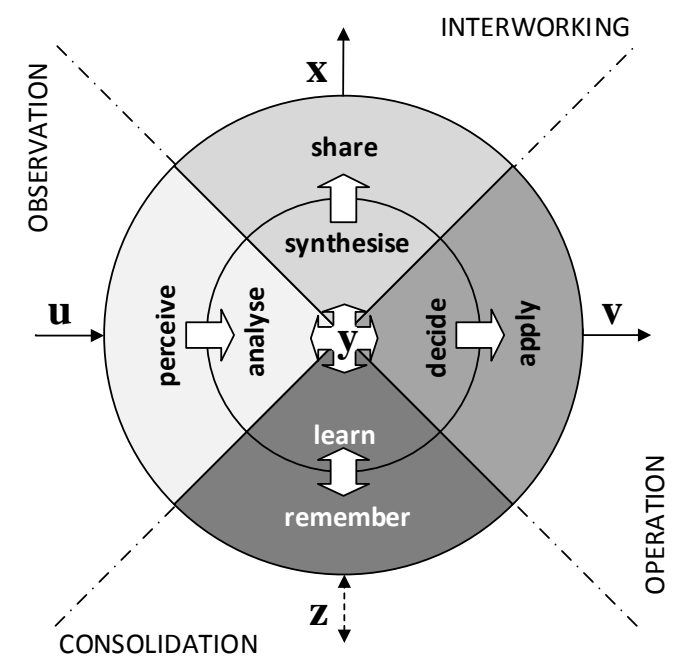

Figure 3: Model for a networked cognitive entity. Revised from (Celentano, 2014).

The phases are respectively linked across the cognitive frontier and the metacognitive hub, as summarised in Table 1.

We can say that the cognitive entity has observable information $\mathbf{u}$ that is used to store knowledge $\mathbf{z}$ and operate instructions $\mathbf{v}$. Instructions, i.e., information, knowledge or commands $\mathbf{x}$, can be externalised. All the above $-\mathbf{u}, \mathbf{v}, \mathbf{z}$ and $\mathbf{x}-$ are at the border of the cognitive entity. The internal steps, occurring at the boundary between the cognitive frontier and the metacognitive hub, imply the presence of, respectively, perceived information $\tilde{\mathbf{u}}$, learnt knowledge $\tilde{\mathbf{z}}$, decision $\tilde{\mathbf{v}}$ and synthesised instructions $\tilde{\mathbf{x}}$. Everything within the core of the metacognitive hub contributes to the internal state $\mathbf{y}$ of the cognitive entity. Fig. 3 illustrates the above notation with the model. The nature of the modelled system has an impact on the architecture of the cognitive frontier.

The interaction of a cognitive entity with other entities or its environment occurs with different directions in different phases, which has an impact on the realisation of those phases in a cognitive device. The cognitive entity gathers inputs or stimuli from an outside entity or the environment, in the perceive phase, whereas it actuates taken decisions in the apply phase. In the share phase, a cognitive entity communicates instructions, i.e., information, knowledge or commands, as needed. In the remember phase, it remembers the learnt or consolidated knowledge and provides both ways access to it. All the above phases possess interfaces to the outside of the cognitive entity. The latter, remember, may not necessarily have 
Table 1: The cognitive and metacognitive phases (left and right half of the table respectively) in the four categories (rows). Revised from (Celentano, 2014).

\begin{tabular}{|l|l|l|l|}
\hline & cognitive frontier & metacognitive hub & \\
\hline perceive & acquiring information & processing information & analyse \\
remember & storing/fetching knowledge & building knowledge & learn \\
share & sharing instructions & preparing instructions & synthesise \\
apply & actuating decisions & generating decisions & decide \\
\hline
\end{tabular}

such, but in general it can. For example, this is needed when access to information is distributed as in clouds, whereas a working memory is likely to be local to the entity. The four outer phases above represent the interfaces towards the entity's outside world, the cognitive frontier. Those interfaces are used as inputs and outputs for commands, ports for communication and data storage and retrieval.

While the outer phases of each category exchange information with their corresponding inner phases only, the four inner phases exchange their own inputs and outputs among themselves, so they form what we call the metacognitive hub. In the analyse phase, inputs are filtered, converted and analysed. Conversely, in synthesise, instructions are selected and adapted for the specific sharing use. So, in the learn phase, the cognitive entity processes commands (actions are not directly observable at metacognitive hub, but decisions are) and information (including the feedback from actions, when available), to build knowledge. Learning may also be exploited for pre-processing incoming stimuli or process instructions for a specific sharing scope. All the above is exploited to take decisions (including prioritisation and planning of actions) in the decide phase.

The generic model described here incorporates the functionalities of a cognitive entity. Clearly, in a given cognitive entity, only a subset of those may be present (or, equivalently, be active).

\section{INTERACTING COGNITIVE ROBOTS}

This section illustrates how the model presented in Sect. 5 is combined into a generic scenario incorporating those is Sect. 4.

Fig. 4 depicts a scenario in which the interaction follows an act-and-observe (Sect. 4) model. The robot at the bottom senses the actuations of the robots at the top of the figure. In this example, the first robot may consolidate the related knowledge for (possibly shared) further use.

Fig. 5 depicts a scenario in which the interaction follows a share-and-act (Sect. 4) model. The robots

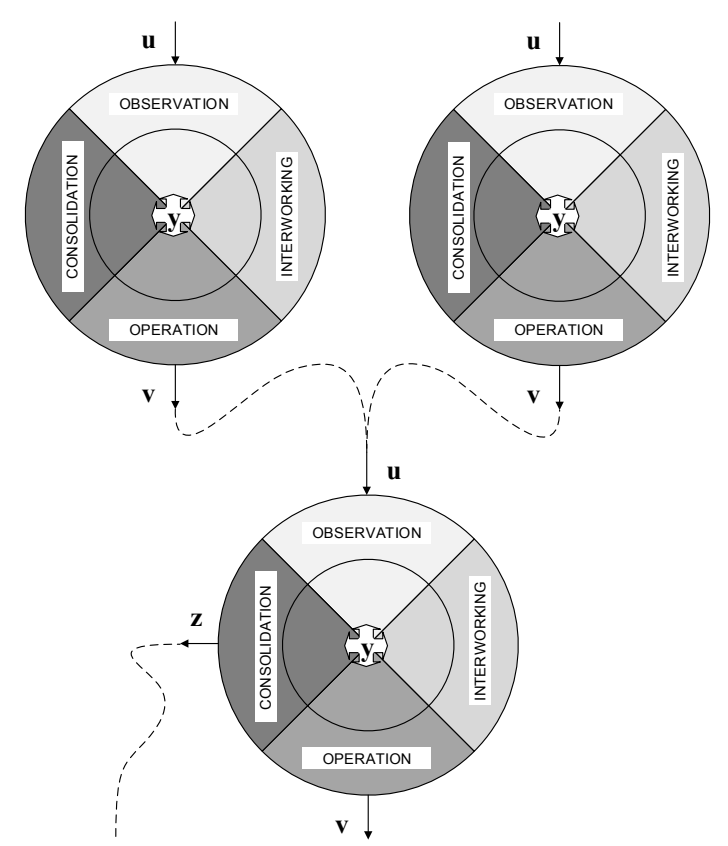

Figure 4: A robot (bottom) sensing actuations of other robots (top).

at the bottom acquire the instructions shared by the robot at the top of the figure. Here, the latter robot gathers before that additional information from the consolidated knowledge.

\section{EVALUATION}

Some related models have been mentioned in Sect. 2 . A thorough collation of the proposed model with related ones is given in (Celentano, 2014) and it is not repeated here, but the key points are summarised in the following.

Cognitive entity models can be assigned to different classes, depending on the relation among their cognitive functions or phases (Celentano, 2014):

A. strictly cycle-based models (phases are visited according to a pre-defined sequence) (Shewhart, 1939; Polya, 1957; Nilsson, 1984; Dobson et al., 


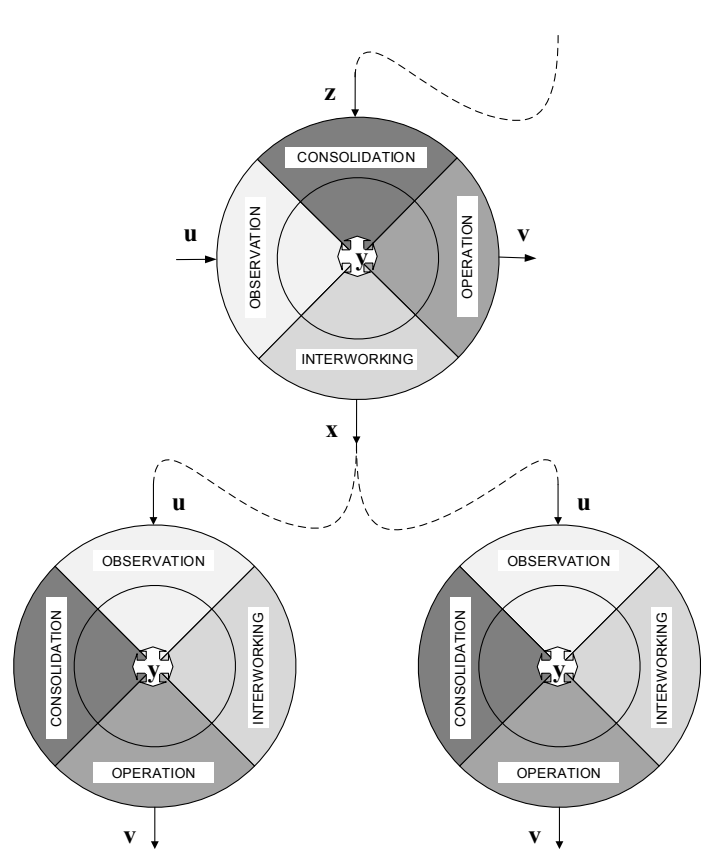

Figure 5: Robots (bottom) acquiring the instructions shared by another robot (top).

2006);

B. nested loops or parallel loops (Bryant, 2004);

C. cycle-based models with a shared phase (all phases except one are visited according to a predefined sequence, but they are connected to a shared phase): (Kephart and Chess, 2003; Albus, 1991);

D. branched cycles with a shared phase (a cycle is somehow defined but short-cuts can be taken) (Mitola, 2000);

E. purely phase-based models (phases are visited with a pattern depending on a specific instantiation or condition) (Anderson et al., 2004; Langley et al., 1987; Kieras and Meyer, 1997; Artz and Armour-Thomas, 1992; Celentano, 2014).

Models with a pre-defined visiting sequence (A, B, C) are less flexible in their applicability over a wider range of cases, which is conversely better for models allowing more free dynamics $(\mathrm{D}, \mathrm{E})$. The proposed model belongs to the latter class $\mathrm{E}$.

Looking into the peculiarities of the above phases, the cognitive functions in an intelligent entity can be categorised as (Celentano, 2014):

1. collecting the inputs;

2. evaluating the inputs;

3. interworking (sharing instructions);
4. decision-making (including planning);

5. implementing the decisions;

6. verifying and generalising the lessons learnt.

As noted earlier, one notable novelty of the proposed model is the explicit presence of the phases collectively labelled above with 3 . The explicit presence in the model of those functions is important since they are related to the specification of the (natural) interfaces involved in social interaction.

The importance of social interaction has been discussed in the above examples (Sect. 4 and 6).

\section{DISCUSSION}

Already in the case of an isolated intelligent entity, self-awareness is crucial for a more conscious execution of tasks. In the case of flocks or swarms, selfawareness improves the behaviour of the entire group. In fact, in those cases it is important to discriminate what I am doing from what the others are doing, even if the final outcome (e.g., relative positions) may look similar. Self-awareness is a pre-requisite for a more effective self-organisation. Self-awareness may exploit explicit and/or implicit interaction among entities.

The model presented in the previous Sect. 5 specifies cognitive phases belonging to interacting cognitive entities, together with the interfaces related to those cognitive functions. Those functions may be realised by separate and integrated element, therefore it makes sense to bring these specifications to established or standardised frameworks, such as ROS or SISO.

The cognitive phases of the present model can be realised as concurrent processes similarly as in the ROS architecture. In particular, the share phase of the model presented in this article is used to communicate instructions before the apply and sometimes even before the decide phase).

Instructions in the model of Sect. 5 correspond to orders, reports and requests in BML. Due to its properties of unambiguity and applicability to international environments, BML is a possible candidate for robotic applications also in other more general cases such as civilian scenarios, including industry environments. Extensions of it might be needed, though, also to let it cope with such diverse situations. 


\section{CONCLUSION}

This article discussed some fundamental issues concerning multi-entity intelligent systems. Particular attention has been given to the observation and related decision-making for system control and examples for multi-robot systems have been discussed. The major means for the needed interaction of robots are the sensing of actuation of other robots, and the coordination based on sharing and acquisition of relevant instructions. The latter implies the possibility of interaction beyond the simplest actuator-sensor acquisition of information.

A cognitive mobile robot should therefore explicitly include such interworking capabilities, which are not found in present models for artificial entities. The model presented in this article fills this gap. Illustrative examples show the role the cognitive phases of this model have in the above two classes of multirobot social interaction. Specifically, this article discussed with the aid of examples how self-awareness can be exploited for self-organisation by detecting and managing behavioural anomalies, i.e., abnormalities in group dynamics.

The cognitive phases of the present model are inspired by both artificial and natural cognitive entities. This makes such a holistic model suitable for use not only for machine-machine, but also for humanmachine interaction.

Interoperability across diverse operational domains and system design reuse across different application domains are both timely topics, and for that robust specifications of functionalities and interfaces to suit the above goals are needed. This article aims at this target.

It is evident that explicitly considering social interaction in the model for an intelligent entity brings advantages to flexible design and specifications. A comparative evaluation of the proposed model has been given here. The evaluation of the concepts discussed in this article by implementation into mobile robots is part of our future work in this area.

\section{ACKNOWLEDGEMENTS}

The authors would like to thank Infotech Oulu for the financial support.

\section{REFERENCES}

Albus, J. (1991). Outline for a theory of intelligence. IEEE Trans. Syst. Man Cybernet., 21(3):473-509.

Anderson, J., Bothell, D., Byrne, M., Douglass, S., Lebiere, C., and Qin, Y. (2004). An integrated theory of the mind. Psychol. Rev., 111(4):1036-1060.

Artz, A. and Armour-Thomas, E. (1992). Development of a cognitive-metacognitive framework for protocol analysis of mathematical problem solving in small groups. Cognition and Instruction, 9(2):137-175.

Brooks, R. (1986). A robust layered control system for a mobile robot. IEEE J. Robot. Autom., RA-2(1):14-23.

Bryant, D. (2004). Modernizing our cognitive model. In Command Control Res. Technol. Symp., pages 1-14, San Diego, CA, US.

Celentano, U. (2014). Dependable cognitive wireless networking: Modelling and design. Number 488. Acta Universitatis Ouluensis Series C: Technica.

Celentano, U. and Röning, J. (ms). Modelling interactive cognitive entities. Manuscript.

Dobson, S., Denazis, S., Fernández, A., Gaiti, D., Gelenbe, E., Massacci, F., Nixon, P., Saffre, F., Schmidt, N., and Zambonelli, F. (2006). A survey of autonomic communications. ACM Trans. Autonomous Adapt. Syst., 1(2):223-259.

Dupourqué, V. (1984). A robot operating system. In IEEE Int. Conf. Robot. Autom., volume 1, Atlanta, GA, US.

Kephart, J. and Chess, D. (2003). The vision of autonomic computing. IEEE Comput., 36(1):41-50.

Kerr, J. and Nickels, K. (2012). Robot operating systems: Bridging the gap between human and robot. In Southeastern Symp. Syst. Theory (SSST), pages 99-104, Jacksonville, FL, US.

Kieras, D. and Meyer, D. (1997). An overview of the EPIC architecture for cognition and performance with application to human-computer interaction. Hum.-Comput. Interact., 12:391-438.

La, H., Lim, R., and Sheng, W. (2015). Multirobot cooperative learning for predator avoidance. IEEE Trans. Control Syst. Technol., 23(1):52-63.

Langley, P., Laird, J., and Rogers, S. (2009). Cognitive architectures: Research issues and challenges. Cogn. Sys. Res., (10):141-160.

Langley, P., Newell, A., and Rosenbloom, P. (1987). Soar: An architecture for general intelligence. Artif. Intell., 33(1):1-64

Mäenpää, T., Tikanmäki, A., Riekki, J., and Röning, J. (2004). A distributed architecture for executing complex tasks with multiple robots. In IEEE Int. Conf. Robot. Autom. (ICRA), pages 3449-3455, New Orleans, LA, US.

Mitola, J. (2000). Cognitive radio: An integrated agent architecture for software defined radio. Kungliga Tekniska Högskola (KTH), Stockholm.

Nilsson, N., editor (1984). Shakey the robot. Number Technical Note 323. Artificial Intelligence Center, SRI International, Menlo Park, CA. 
Peschl, M., Röning, J., and Link, N. (2012). Human integration in task-driven flexible manufacturing systems. In Katalinic, B., editor, Ann. DAAAM 2012 \& Proc. 23rd Int. DAAAM Symp., pages 85-88, Zadar, Croatia.

Polya, G. (1957). How to solve it: A new aspect of mathematical method. Doubleday Anchor Books, Garden City, NY, 2nd edition.

Quigley, M., Gerkey, B., Conley, K., Faust, J., Foote, T., Leibs, J., Berger, E., Wheeler, R., and Ng, A. (2009). ROS: an open-source robot operating system. In ICRA Workshop Open Source Software, Kobe, Japan.

Rein, K., Schade, U., and Hieb, M. (2009). Battle management language (BML) as an enabler. In IEEE Int. Conf. Commun. (ICC), Dresden, Germany.

Remmersmann, T., Brüggemann, B., and Miłosław, F. (2010). Robots to the ground. In Mil. Commun. Inform. Systems Conf. (MCC), pages 61-68, Warsaw, Poland.

Shewhart, W. (1939). Statistical method from the viewpoint of quality control. Graduate School of the Department of Agriculture, Washington, DC. Reprint by Dover Publications, Mineola, NY, 1986.

Thomas, R., DaSilva, L. A., and MacKenzie, A. (2005). Cognitive networks. In IEEE Int. Symp. New Frontiers Dynam. Spectrum Access Netw. (DySPAN), pages 352-360, Baltimore, MD, US. 